\title{
Ultrasonic/Sonic Sampler and Sensor Platform for In-situ Planetary Exploration
}

\author{
Y. Bar-Cohen, S. Sherrit, X. Bao, and Z. Chang \\ Jet Propulsion Laboratory/Caltech, Pasadena, California, USA \\ E-mail yosi@jpl.nasa.gov Web: http://ndeaa.jpl.nasa.gov
}

\begin{abstract}
The search for existing or past life in the Universe is one of the most important objectives of NASA's mission. In support of this objective, an ultrasonic/sonic mechanism is currently being developed at JPL to allow probing and sampling of rocks and to use the mechanism as a sensor platform for in-situ planetary analysis. The technology is based on the novel Ultrasonic/Sonic Driller/Corer (USDC), which requires low axial force, thereby overcoming one of the major limitations of planetary sampling using conventional drills in low gravity environments. The USDC was demonstrated to: 1) drill ice and various rocks including granite, diorite, basalt and limestone, 2) not require bit sharpening, 3) operate at low and high temperatures, and 4) operate at low average power using duty cycling.. The capabilities that are being investigated include probing the ground to select sampling sites, collecting various forms of samples, and hosting sensors for measuring various properties. A series of modifications of the USDC basic configuration were implemented including an ultrasonic abrasion tool (URAT), Ultrasonic Gopher for deep drilling, and the Lab-on-a-drill. These modifications will be described in this paper.
\end{abstract}

Keywords: USDC, Ultrasonic/Sonic Drill/Corer, URAT, Lab-on-a-drill

\section{Introduction}

NASA exploration missions increasingly are required to perform sampling, in-situ analysis and possibly the return of sample to Earth for further tests. Existing drilling techniques are limited by the need for large axial forces and holding torques, high power consumption and an inability to efficiently duty cycle. Lightweight robots and rovers have difficulties accommodating these requirements. To address these limitations the ultrasonic/sonic driller/corer (USDC) was developed [Bao, et al, 2003; and BarCohen et al, 2001, Sherrit, et al, 1999; \& 2000; $\quad$ http://ndeaa.jpl.nasa.gov/nasande/usdc/usdc.htm;]. The actuator of the USDC consists of a piezoelectric stack with backing and a horn for the amplification of the induced displacement. The actuator is driven in resonance at $20-\mathrm{kHz}$ and a stress bolt holds the stack in compression to prevent fracture during operation. Unlike typical ultrasonic drill where the drill bit is acoustically coupled to the horn, in the USDC the actuator drives a free flying mass (free-mass), which bounces between the horn tip and the drilling or coring bit at sonic frequencies. The impacts of the freemass create stress pulses that propagate to the interface of the bit and the rock onto which the USDC is placed in contact. The rock fractures when its ultimate strain is exceeded at the rock/bit interface. The low mass of a USDC device and the ability to operate with minimum axial load with near zero torque (see Figure 1) offers important capabilities for sample acquisition and insitu analysis. Another important 
characteristic of the USDC is the capability to operate in the harsh environments of space including low or high temperature and/or pressure.
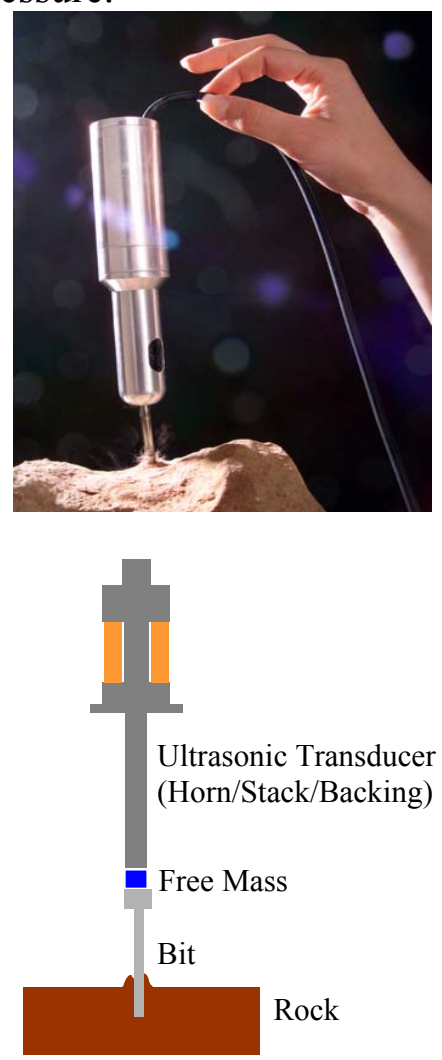

FIGURE 1: The USDC is shown coring with minimum axial force and holding torque (top), and a schematic diagram of the USDC device (bottom).

The USDC has been demonstrated to drill rocks that range in hardness from granite and basalt to sandstone and tuff. Other media that have been drilled include soil, ice and diorite, and limestone. This novel drill is capable of high-speed drilling (2 to $20-\mathrm{mm} / \mathrm{Watt} \cdot \mathrm{hr}$ for a $2.85 \mathrm{~mm}$ diameter bit) in basalt and Bishop Tuff using low axial preload $(<10 \mathrm{~N})$ and low average power $(<5 \mathrm{~W})$. It drilled 25-mm deep, 6-mm diameter holes in basalt in a little over 2-hrs from a 4-kg platform using $10 \mathrm{~W}$ average and $25 \mathrm{~W}$ peak power. Also, it has drilled 15-cm deep, 5-mm diameter holes in sandstone in just over an hour using similar power as for the basalt drilling.

The operation of the USDC was analytically modeled to allow effective design. This model examined the USDC as a system with five elements including the electrical driver, ultrasonic transducer, freemass, drill stem, and the rock. In the initial modeling the main elements and the interaction between them were analyzed and modeled separately. A one-dimensional model was then developed for each interaction (except the bit-rock interaction) and an integrated software program was developed to simulate the operation of all parts of the USDC. The strain that is induced in the rock was calculated by using a two-dimensional axisymmetric model and the drilling rate was estimated based on the specific energy required to fracture the rock

As a lab-on-a-drill, currently the USDC is being considered for probing sampled materials and the surroundings area prior to acquisition in order to optimize the selection of sites with the highest likelihood of containing biological signatures. Methods of acquiring samples in different forms are being studied and the effect of the sampling process is investigated to assure minimum impact on the sample characteristics. Using sensors that are mounted on the bit, including thermocouple and fiberoptic, real time measurements are enabled reducing concern of cross contamination that may result from sample handling.

\section{Comparison of USDC with Conventional Drilling Techniques}

The USDC ability to drill using low axial load created new options for the exploration of low gravity bodies in the universe (e.g., comets and asteroids). A comparison between the USDC and conventional drills is given in Table 1. Unlike conventional drills, the USDC can drill or core rocks from 
very light and flexible platforms (e.g., Sojourner or Marie Curie rovers, robotic arms, etc.). Since the USDC does not have electrical motors, it can be duty cycled without significant loss of efficiency. This facilitates operations under very low average power (operation at 2-4 watts average power has been demonstrated). Unlike conventional drills, the drive mechanism of the USDC has only three moving parts, which are not physically connected and do not require lubricants. This design eliminates common mechanical failure modes and makes it easy to constrain during launch. The use of a piezoelectric stack as the USDC actuator permits the device to operate over a very wide temperature range. The drilling/coring bit does not need to rotate and does not require sharpening. The USDC can core arbitrary cross-sections (square, round, hexagons) and can accommodate drilling of vertical and overhanging rock faces.

TABLE 1: Comparison between the USDC and conventional Drills

\begin{tabular}{|c|c|c|}
\hline & Conventional Drills & USDC \\
\hline Axial preload & $>100 \mathrm{~N}$ (typically $150 \mathrm{~N}$ ) & $<10 \mathrm{~N}$ \\
\hline $\begin{array}{l}\text { Drill walk at } \\
\text { core initiation }\end{array}$ & $\begin{array}{l}>30 \mathrm{~N} \cdot \mathrm{m} \text { induced torques and } \\
>100 \mathrm{~N} \text { tangential forces }\end{array}$ & $<1 \mathrm{~N}$ \\
\hline $\begin{array}{l}\text { Average power } \\
\text { to create } 10-\mathrm{mm} \\
\text { core }\end{array}$ & $\begin{array}{l}>20-30 \mathrm{~W} \text {. Can be reduced but the drilling } \\
\text { efficiency goes down. }\end{array}$ & $\begin{array}{l}\text { Can be as low as 2- } \\
3 \mathrm{~W} \text { (lower power } \\
\text { requires longer } \\
\text { drilling) }\end{array}$ \\
\hline Duty cycling & Involve staggering loss of efficiency & $\begin{array}{l}\text { Very little efficiency } \\
\text { loss ( } 2 \mathrm{~W} \text { average at } \\
25 \mathrm{~W} \text { peak was } \\
\text { demonstrated) }\end{array}$ \\
\hline $\begin{array}{l}\text { Current } \\
\text { Overshoot }\end{array}$ & $\begin{array}{l}\text { 3-4 times larger startup electrical currents than } \\
\text { those during continuous operations }\end{array}$ & $\begin{array}{l}<20 \% \text { even with duty } \\
\text { cycling. }\end{array}$ \\
\hline Drill chatter & $\begin{array}{l}\text { Induces low frequency }(2-10 \mathrm{~Hz}) \text { and high force } \\
\text { perturbations on the drilling platform }\end{array}$ & Minimal \\
\hline Support system & $\begin{array}{l}\text { Requires stable and massive platforms with solid } \\
\text { anchoring }\end{array}$ & Minimal \\
\hline $\begin{array}{l}\text { Drilling/Coring } \\
\text { soft rock }\end{array}$ & Shearing and spalling & Compression failure \\
\hline $\begin{array}{l}\text { Drilling/Coring } \\
\text { hard rocks }\end{array}$ & $\begin{array}{l}\text { Grinding with corresponding } 300 \% \text { increase in } \\
\text { energy consumed per unit volume of removed } \\
\text { rock. Require frequent sharpening or replacement. } \\
\text { Otherwise, } 10 \text { fold increase in heat generation and } \\
\text { similar drop in efficiency. }\end{array}$ & $\begin{array}{ll}\text { - } & \text { Spalling } \\
\text { - } & \text { No need for drill } \\
\text { bit sharpening }\end{array}$ \\
\hline
\end{tabular}

The simplicity of the USDC based sampler reduces the number of possible contamination sources (e.g., lubricants and metallic filings from wear on the gears). The acoustic vibration of the bit provides for transport of the powdered cuttings away from the bit/sample interface. This design minimizes cutting edge wear and prevents particle entrapment unlike conventional composite drilling bits (e.g., diamond cutters on silicon carbide substrate with metal holders). The USDC can also use bits 
manufactured from a single element (e.g. Tungsten) and could thereby minimize the sources of trace metallic and abrasive elements.

Non-traditional (or "modern" in the oil industry terminology) drilling technologies (laser, electron beam, microwave, jet, etc.) usually are competitive only in applications that are time limited (time is money in oil industry) and not power/mass limited as is typical for the current space science applications. Typically, the down-the-well energy that is required to remove a unit volume of rock for "modern" technologies is the same as grinding and melting. The required energy is 3 and 5 times higher, correspondingly, than that for shear drilling. Generally, the ratio of power delivered down-the-well vs. input power generation for these non-traditional approaches is below several percent vs. $10 \%-30 \%$ for conventional drills (comparable to USDC). Consequently, many space missions do not have enough power to employ these "modern" drilling technologies.

\section{Modifications of the USDC}

Various novel modifications were investigated to determine the possible mechanisms and devices that can be developed in support of the NASA exploration of various planets. Several devices were developed and demonstrated including the lab-on-a-drill, the ultrasonic rock abrasion tool (URAT) and the ultrasonic gopher.

\subsection{Lab-on-a-drill}

As mentioned above, the ultrasonic/sonic driller/corer (USDC) allows for both sampling and probing the sampled medium while it can carry sensors for in-situ analysis. These integrated system capacities received the moniker lab-on-a-drill. Vibrations, which are generated by the device upon impacting an object or the ground, induce elastic waves and provide a sounding mechanism for noninvasive probing of the impacted medium. This sounding capability combined with detectors can provide information about ground subsurface-layered structure, mechanical properties, and presence of geological cavities. Further, pressing the device onto various materials or objects modifies the mechanical impedance of the piezoelectric stack that actuates the USDC. Gauging the changes in the electrical impedance can provide information for estimating the stiffness of the pressed medium. These two probing capabilities allow screening objects prior to drilling or coring them. Also, equipping the bit with sensor such as fiberoptic or thermocouples allows real-time on-board or remote analysis. In addition to probing and sensing, the USDC can be designed to acquire various forms of samples for on-board or post analysis and other applications. The types of samples that can be extracted include cores, and powdered cuttings. The sampled material can be collected from the front of the device, through the hollow center or through various tubing that are connected to the device assembly.

Coring via the USDC mechanism involves rock fracture under impact loading (percussion). To better understand the fracture of rocks under this impact loading, a finite element model using ANSYS (a finite element software package) was developed to investigate the propagation of the induced stress. Results were derived by assuming that the rock medium is made of isotropic homogeneous material with a Young's modulus of $10 \mathrm{GPa}$ and Poisson's ratio of 0.3. Examples of data for various soil and rock types and the related range of Young's modulus are listed in Table 2. Contour maps of the maximum principal strain were plotted and used to indicate the areas where the rock is fractured and to determine how 
the elastic waves propagate in the rock prior to fracture. This analytical capability allows for an estimate of the limitations on the diameter of the cored material that maintains structural integrity. Using this analysis, it is estimated that the minimum diameter for an intact core of a homogeneous material that can be produced is about $4-5 \mathrm{~mm}$ for medium to hard materials. Also, the drilling rates were calculated for drilling various rocks via $10-\mathrm{W}$ average power (see Figure 2).

\begin{tabular}{|l|l|}
\hline $\begin{array}{c}\text { Soil conditions and/or } \\
\text { Rock Type }\end{array}$ & $\begin{array}{c}\text { Young's } \\
\text { Modulus }\end{array}$ \\
\hline Loose sand & $10-25 \mathrm{MPa}$ \\
\hline Medium dense sand & $20-60 \mathrm{MPa}$ \\
\hline Dense sand & $50-100 \mathrm{MPa}$ \\
\hline Sedimentary sandstone & $10-60 \mathrm{GPa}$ \\
\hline Igneous Basalt & $60-80 \mathrm{GPa}$ \\
\hline Sedimentary limestone & $60-80 \mathrm{GPa}$ \\
\hline Igneous Anorthosite & $83 \mathrm{GPa}$ \\
\hline
\end{tabular}

TABLE 2: Typical values of the elastic modulus for soils and rocks sense the elastic waves that are imparted into the ground and to support analysis of the received wave characteristics. As a diagnostic tool, this probing method can provide information about the soil mechanical properties, geological anisotropy, and layered characteristics as well as detect, locate and characterize geological cavities. The presence of layers causes dispersive wave characteristics, small discontinuities cause scattering and attenuation, and large discontinuities (rocks,

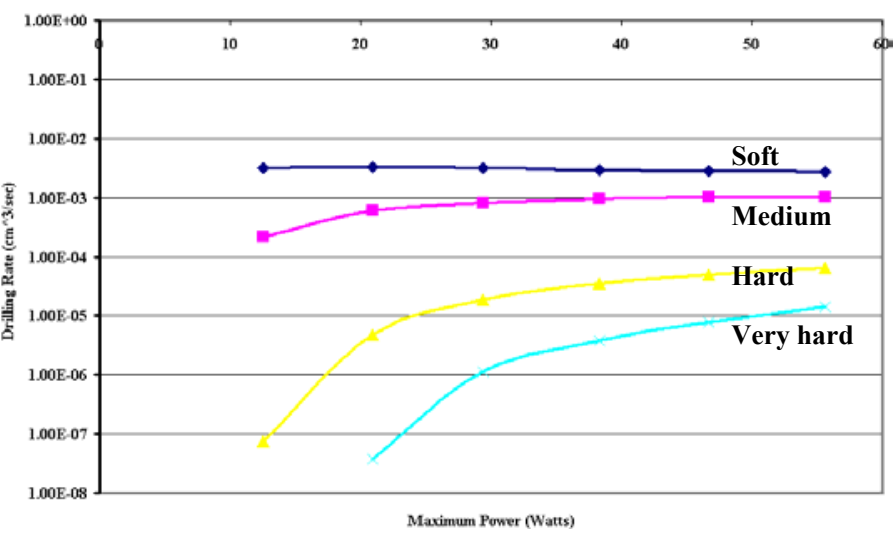

FIGURE 2: Analytical drilling rates in rocks with various hardness levels using $10 \mathrm{~W}$ average power. The rocks are classified by their compression strength, where: Soft: $0-50$; Medium: $50-$ 100; Hard: 100 - 200; and Very hard: >200 (MPa).

\subsubsection{Noninvasive Geophysical Probing}

Generally, the use of noninvasive methods to determine the mechanical properties of the ground is an outgrowth of the field of seismology. These methods transmit elastic waves through a medium and analyze the received waves after interacting with the various geophysical features, layer characteristics, material/ground physical properties and flaws in the host media. The induced hammering action from a USDC provides a sounding mechanism for noninvasive probing of the ground geology to provide information about its subsurface structure, and mechanical properties [Chang, et al, 2003]. To take advantage of this possibility, accelerometers can be used to cavities, etc.) produce reflections. Methods are available to extract geophysical information from the imparted waves [Nazarian, et al, 1993 \& 1997], offering effective probing and imaging for in situ geophysical probing providing valuable information about the stiffness constants of soil and rocks. As can be seen from Table 2, the elastic moduli of soils and rocks have distinctive ranges that vary in orders of magnitudes. So far studies of surface wave velocity for various rocks have shown quantitative data that is repeatable but did not seem to strongly correlate with rocks hardness and more effort is underway to determine the applicability of other wave modes. 


\subsubsection{Sampling Rocks and Powdered Cuttings}

The USDC is capable of producing samples in the form of cores and powdered cuttings. In order to acquire this sampled material there was a need to modify the bit to accommodate the specific requirements of the sampled material. To extract the produced cores a novel bit design as shown in Figure 3 was implemented in the form of an all-in-one bit. This bit is designed to core, fracture the core at the bottom when reaching the desired length, and retain the acquired core while allowing for the release of the core at the selected caching location. The bit was constructed with a wedge at the top of the inner surface and a set of springs were imbedded into the sidewalls. The wedge introduces transverse forces at the top of the core to cause maximum stress near the root and shear fractures the core. This mechanism was demonstrated experimentally in several rock samples. The side spring was introduced as a means of retaining the produced core until it is delivered to the caching location. A hole along the center of the bit was introduced to allow using a pushrod to push the core into the caching location.

In order to extract the powdered cuttings that are formed during drilling a set of concentric tubes were used. A pressurized $\mathrm{CO}_{2}$ was blown thru the gap between the tubes and the mixture of the gas with the powder was funneled through the center tube along the hollow core of the USDC to the desired location away from the USDC.

\subsubsection{USDC with integrated sensor suite}

Since the USDC bit does not turn and its vibration amplitude is relatively small, one can easily integrate sensors into the bit and conduct real-time tests during sampling. To date, two types of sensors were successfully demonstrated: thermocouple and fiberoptic. The thermocouple was used to measure the rate and maximum rise of temperature and the data was shown to correlate quite nicely with the hardness of the rocks that were drilled. Even though these thermal changes are dependent on the heat conductivity and capacity of the drilled object, the majority of the rocks tested have relatively similar thermal properties. It is expected that when measuring high $\mathrm{Fe}$ content rocks such as magnetite that this relationship between hardness and temperature rise would fail. Compiling temperature rise rate and maxima as a function of time for variety of drilled materials has demonstrated the feasibility of using a thermocouple-on-the-bit as a means of assessing the drilled medium hardness (see Figure 3).

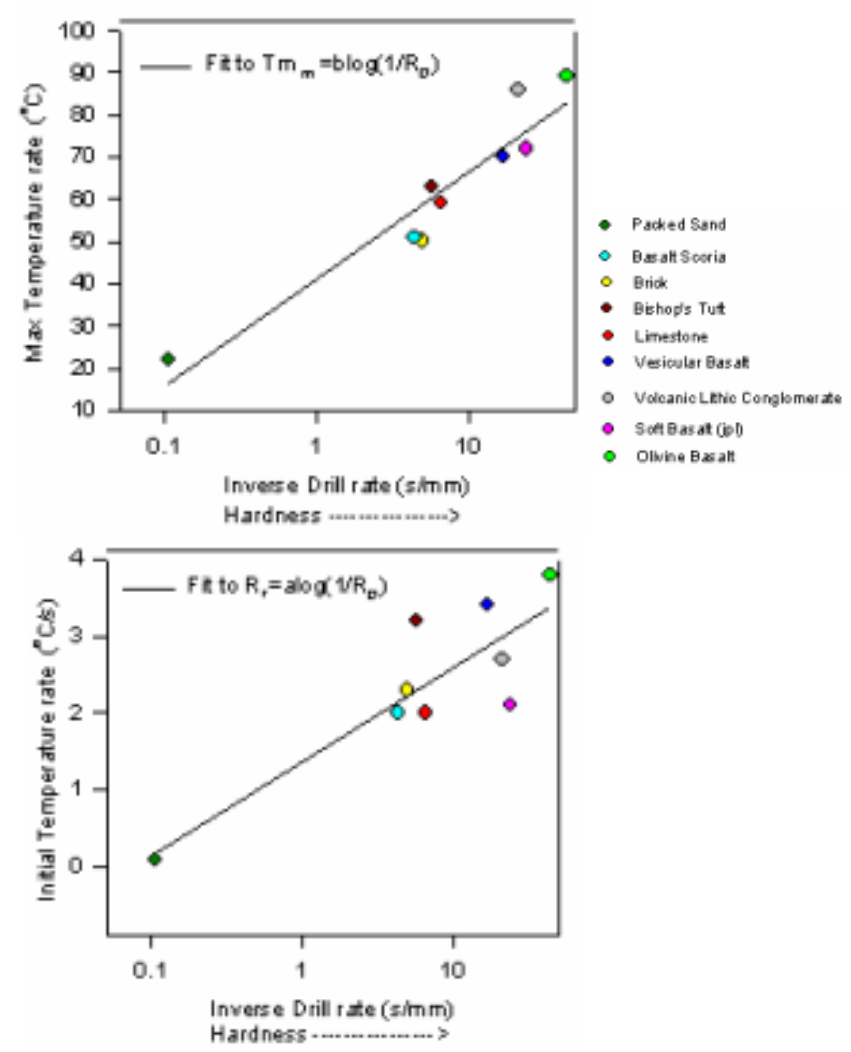

FIGURE 3: The measured temperature maxima and rise rate as a function of hardness (inverse drilling rate) for variety of media. 


\subsection{Ultrasonic rock abrasion tool (URAT)}

Generally, The surface of Mars is subjected to various effects including weathering, impact of meteorites and erosion. In order to obtain useful information about Mars planetary geologists and astrobiologists are seeking to remove the outer layers $(5 \mathrm{~mm})$ from the sampled rock to get access to pristine material. To address this need the USDC was modified to operate as an abrader, similar to a meat tenderizer, and to demonstrate the concept the logo of JPL was engraved on a basalt rock (Figure 4). These letters were carved in approximately 40 minutes using a tungsten carbide coated sphere and a power of 12 watts. Using this mechanism an Ultrasonic Rock Abrasion Tool (URAT) was developed and successfully demonstrated to abrade the same basalt rock. A photograph of the URAT is shown in Figure 5. along with a cut-sectional view of a 3D model. The device was designed and produced as a backup tool for the Mars '03 mission and it was successfully demonstrated to meet the required mission objectives. The URAT's bit was made of a $4 \mathrm{~cm}$ diameter disk with 4-mm spaced pyramidal shape risers (emulating a meat tenderizer) that when actuated hammered the rock surface and abraded its top layer. An example of an abraded area on basalt is shown in Figure 4.

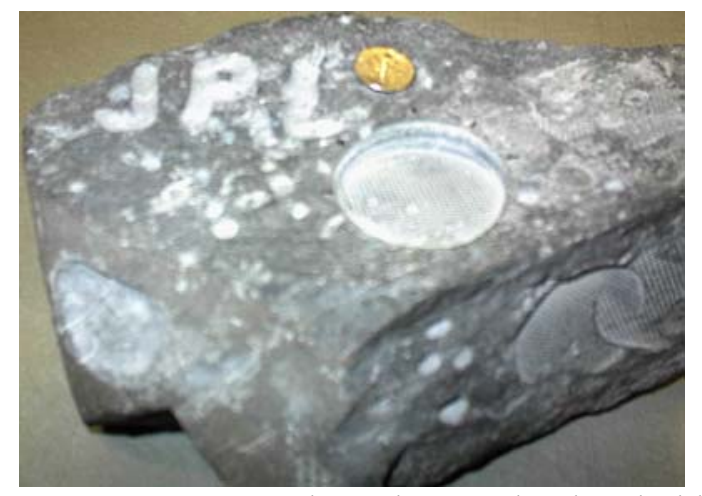

FIGURE 4: Basalt rock sample abraded by the ultrasonic rock abrasion tool.
The complete device weighs $0.4-\mathrm{kg}$ and has the dimensions: length $-14.4 \mathrm{~cm}$; largest diameter - $6.35-\mathrm{cm}$; and volume - $460-\mathrm{cm} 3$. The piezoelectric stack/horn actuator is housed in a cylindrical container and the movement of the actuator with respect to the housing is aided with mechanical sliders. A dust shield surrounds the bit to form a seal against the rock to reduce rock debris migration into sensitive instrument or mechanical areas. A bushing at the bottom of the housing acts to reduce dust flow into the actuator.

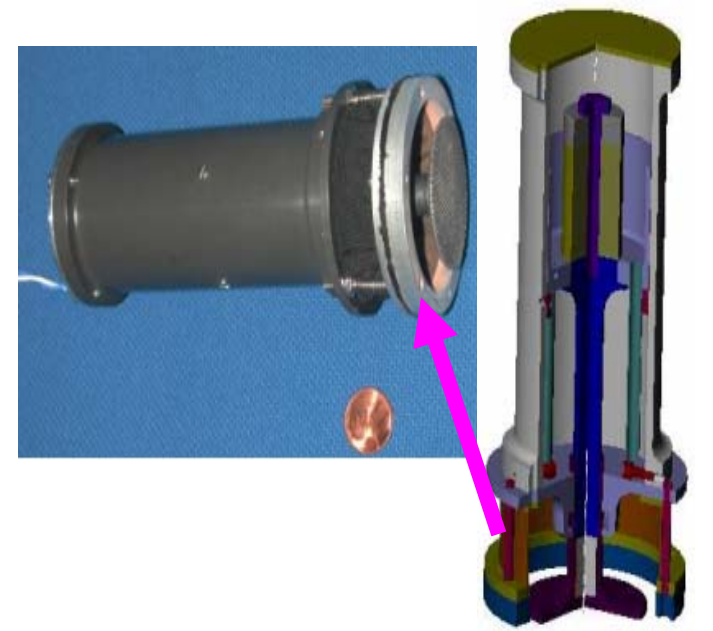

FIGURE 5 Photographic (left) and crosssection graphic view (right) of the URAT

\subsection{Ultrasonic gopher}

In order to address the need to drill several meters deep into the surface of planets with low gravity (Mars $\approx 0.3 \mathrm{~g}$ ), the USDC was modified (in cooperation with Cybersonics, Inc.) to produce the Ultrasonic-Gopher (Figure 6) [Bar-Cohen, et al, 2002]. The device uses a bit that has a diameter ( 3 and $4.5 \mathrm{~cm}$ were demonstrated) that is larger than the actuator. Generally, the bit creates a borehole that is larger than the bit outer diameter and a core that is smaller than the inner diameter of the bit. This reduces the chances of bit jamming when the borehole integrity is maintained, and it eases the 
extraction of the core from the bit. The horn was configured with an inverted geometry and a bobbin-shape integral free-mass was located below the horn to serve also as a connecting element between the horn and the bit. Since the current analytical model suggests that the USDC performance does not change significantly with changes in ambient gravity, the gopher is expected to operate as an effective coring device for extraterrestrial geological bodies.

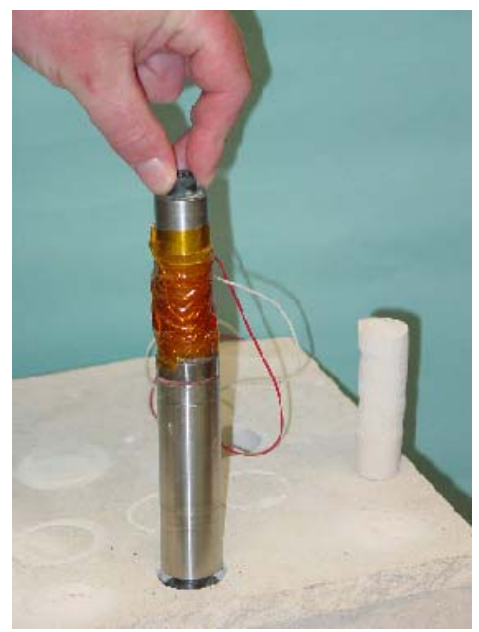

FIGURE 6: An Ultrasonic-Gopher and an extracted core (right) from a limestone.

\section{Operation from various platforms}

Given the low axial force that is needed to drill with the USDC a series of demonstrations were made to show the feasibility of this technology to support exploration missions. To examine its performance from the Sojourner Rover (see Figure 7) a tube was used as a guide through which the USDC was inserted and allowed to operate using the mass of the drill as the load. The tube internal diameter was about $5.0-\mathrm{cm}$, which is larger than the external diameter of USDC. In this configuration, the USDC was easily shown to drill holes and cores in various rocks including sandstone, and others. Also, the USDC was mounted onto the robotic arm of the FIDO rover and was demonstrated to drill with minimal mechanical vibration transferred to the arm (See Figure 8). Further, it was easily used to reinsert the drill bit into the borehole that was drilled earlier. The ability of the USDC to drill under low axial load offers the potential to operate from such platforms as inflatable systems using minimal anchoring and thus enabling capabilities that are not possible with other drilling technologies.

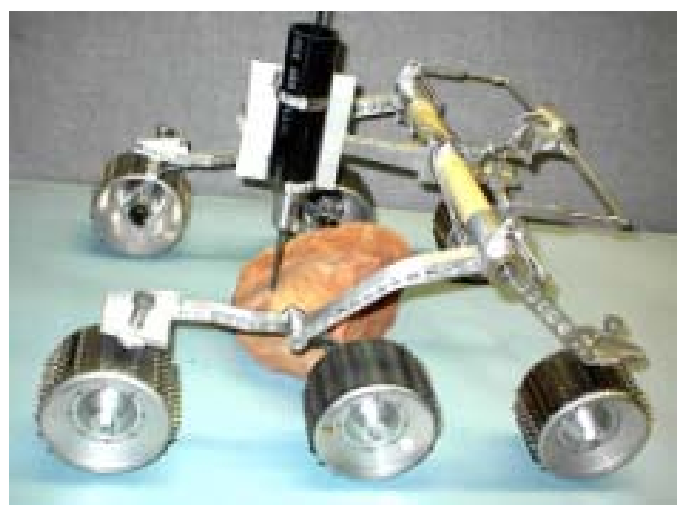

FIGURE 7: The USDC mounted on the Sojourner Rover platform is shown to drill sandstone.

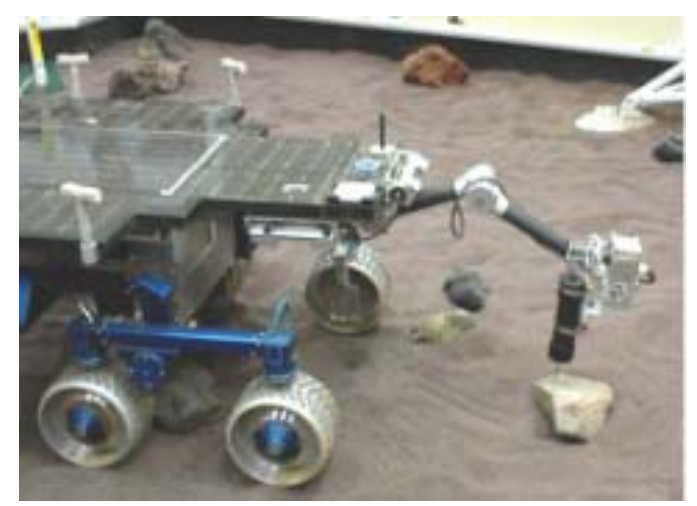

FIGURE 8: The USDC is shown drilling while mounted on the robotic arm of the FIDO Rover.

\section{Summary}

The ultrasonic/sonic driller/corer (USDC) was investigated for various potential 
planetary applications. To allow effective design and construction of the various modifications of the USDC an analytical model was developed to predict its behavior towards the goal of optimizing its performance. The drilling rates calculated from the model were found to be in general agreement with drilling rate data measured on a variety of rock samples. Using a simulation of the USDC operation the performance of the USDC was investigated analytical and the results were used to guide the design of various modifications of the device. Three novel designs were developed and demonstrated including the Lab-on-a-Drill, Ultrasonic Gopher and the URAT. The Lab-on-a-Drill is intended to take advantage of the probing capabilities of the USDC, the capability to sample cores and powdered cuttings as well as the fact that sensors can be easily mounted on the bit and allow real time data acquisition while drilling. The URAT was demonstrated to remove rock layers from rocks that are as hard as Basalt. This device was developed as a backup tool for the mission to Mars in 2003 and successfully met the required objectives. The third modification was in the form of the Ultrasonic Gopher that was developed for deep drilling. This device operates in a cyclic mode of coring, uploading, core caching and downloading. The device is now being investigated for drilling up to 20-meters in ice in Antarctica. Since its first been introduced in 1999, the USDC has continually been advanced and the various novel modifications that were made enabled significant capabilities for future NASA missions.

\section{Acknowledgement}

Research reported in this manuscript was conducted at the Jet Propulsion Laboratory (JPL), California Institute of Technology, under a contract with National Aeronautics Space Agency (NASA).

\section{Reference}

Bao X., Y. Bar-Cohen, Z. Chang, B. P. Dolgin, S. Sherrit, D. S. Pal, S. Du, and T. Peterson, "Modeling and Computer Simulation of Ultrasonic/Sonic Driller/Corer (USDC),"Accepted for publication in IEEE UFFC.

Bar-Cohen Y., S. Sherrit, B. Dolgin, S. Askin, and X. Bao, "Ultrasonic/Sonic Mechanism of Deep Drilling (USMOD)," JPL New Technology Report, submitted on June 12, 2001, Docket No. 30291, July 17, 2001. Patent was submitted on Nov. 23, 2002.

Bar-Cohen Y., S. Sherrit, B. Dolgin, T. Peterson, D. Pal and J. Kroh, "Ultrasonic/Sonic Driller/Corer (USDC) With Integrated Sensors," JPL New Technology Report, Docket No. 20856, Submitted on August 30, 1999, Patent was submitted on May 1, 2001.

Chang Z., S. Sherrit, X. Bao, and Y. BarCohen, "In-situ Rock Probing Using The Ultrasonic/ Sonic Driller/Corer (USDC)," Proceedings of the SPIE Smart Structures and Materials Symposium, Paper 5056-73, San Diego, CA, March 3-6, 2003.

Nazarian S., Baker M.R. and Crain K. "Assessing Quality of Concrete with Wave Propagation Techniques," Materials Journal, American Concrete Institute, Vol. 94, No. 4, Farmington Hills, MI, (1997), pp. 296-306.

Nazarian S., Baker R.B. and Crain K., "Fabrication and Testing of a Seismic Pavement Analyzer," Report H-375, Strategic Highway Research Program, Washington, DC, Dec. 1993, p. 156.

Sherrit S., B. P. Dolgin, Y. Bar-Cohen, D. Pal, J. Kroh, and T. Peterson, "Modeling of Horns for Sonic/Ultrasonic 
Applications," Proceedings of the IEEE

International Ultrasonics Symposium, held in Lake Tahoe, CA, 17-20 October 1999, pp. 647-651.

Sherrit S., X. Bao, Z. Chang, B.P. Dolgin, Y. Bar-Cohen, D. Pal, J. Kroh, and T. Peterson "Modeling of the Ultrasonic/Sonic Driller/Corer (USDC)," Proceedings of the IEEE Ultrasonics Symposium, San Juan, Puerto Rico, Oct 2000. 